\title{
Exact Symbol Error Probability of Square M-QAM Signaling over Generalized Fading Channels subject to Additive Generalized Gaussian Noise ${ }^{\star}$
}

\author{
Hamza Soury, Ferkan Yilmaz, and Mohamed-Slim Alouini \\ Computer, Electrical, and Mathematical Sciences and Engineering (CEMSE) Division \\ King Abdullah University of Science and Technology (KAUST) \\ Thuwal, Makkah Province, Kingdom of Saudi Arabia \\ Email: \{soury.hamza, ferkan.yilmaz, slim.alouini\}@kaust.edu.sa
}

\begin{abstract}
This paper considers the average symbol error probability of square Quadrature Amplitude Modulation (QAM) coherent signaling over flat fading channels subject to additive generalized Gaussian noise. More specifically, a generic closedform expression in terms of the Fox $\mathrm{H}$ function and the bivariate Fox $\mathbf{H}$ function is offered for the extended generalized-K fading case. Simplifications for some special fading distributions such as generalized-K fading, Nakagami- $m$ fading, and Rayleigh fading and special additive noise distributions such as Gaussian and Laplacian noise are then presented. Finally, the mathematical formalism is illustrated by some numerical examples verified by computer based simulations for a variety of fading and additive noise parameters.

Index Terms-Symbol error probability, quadrature amplitude modulation, additive generalized Gaussian noise, generalized Gaussian Q-function, Laplacian noise, generalized composite fading channels, extended generalized-K fading, generalized-K fading, and Nakagami- $m$ fading.
\end{abstract}

\section{INTRODUCTION}

The performance analysis of M-ary Quadrature Amplitude Modulation (M-QAM) signalling over generalized fading channels subject to additive white Gaussian noise (AWGN) has been extensively studied [1]-[3]. However, the additive noise may deviate from the traditional Gaussian distribution and in this case it has been widely reported that the additive noise can be modeled by a generalized Gaussian distribution which includes for instance the Gaussian, Laplacian, and uniform distributions as special cases [4]-[6]

In this paper, we consider a system with an input signal generated according to a square M-QAM constellation fed to a channel with an Extended Generalized-K (EGK) fading envelope [7], and subject to additive white generalized Gaussian noise (AWGGN). The goal of this paper is to find a generic expression for the probability of error of the system under consideration and then to simplify this expression as much as possible for different values of the fading and noise parameters.

The paper is organized as follows. First, a general integral form of the Symbol Error Probability (SEP) using the Generalized Gaussian Q-function [8] is derived in Section II.
Next, a generic closed-form expression specific to the EGK fading is given in Section III. Some special cases with simpler expressions are presented in Section IV. Section V offers some selected numerical results followed by some concluding remarks in Section VI.

\section{Average SEP of SQuARE M-QAM}

Consider a typical single input single output (SISO) communication system. The transmitted signal $\mathcal{S}$ carries symbols mapped according to a square M-QAM constellation, where $\mathrm{M}$ is an even power of $2 . \mathcal{S}$ is first multiplied by a channel fading envelope $\mathcal{H}$ which has a generalized flat fading characteristics and is then corrupted by an AWGGN with zero mean and variance $N_{0} / 2$. More specifically, the probability density function (PDF) of the AWGGN noise is given by [8, Eq. (2)]

$$
p_{\mathcal{N}}(n \mid \mu, \sigma, \alpha)=\frac{\alpha \Lambda}{2 \Gamma(1 / \alpha)} \exp \left(-\Lambda^{\alpha}|n-\mu|^{\alpha}\right),
$$

defined over $n \in \mathbb{R}$, where the parameters $\alpha \in \mathbb{R}^{+}$and $\mu \in$ $\mathbb{R}$ denote the shaping parameter and the mean, respectively. Moreover, the coefficient $\Lambda$, which is related to normalizing the noise power, is defined as

$$
\Lambda=\frac{\Lambda_{0}}{\sigma}=\sqrt{\frac{2 \Gamma(3 / \alpha)}{N_{0} \Gamma(1 / \alpha)}},
$$

where $\Lambda_{0}=\sqrt{\Gamma(3 / \alpha) / \Gamma(1 / \alpha)}$ is the normalizing coefficient with respect to the shaping parameter $\alpha$, and the parameter $\sigma^{2}=\mathbb{E}\left[\mathcal{N}^{2}\right]-\mu^{2}=N_{0} / 2$ denotes the variance of the AWGGN noise. Furthermore, in (1) (i.e., also seen in (2)), $\Gamma($.$) denotes the Gamma function. Note that the Laplacian$ noise is obtained for $\alpha=1$, the Gaussian noise for $\alpha=2$, and the uniform noise for $\alpha=\infty$.

The performance analysis of the system above is well studied in the Gaussian noise case, and for a given value of the signal to noise ratio (SNR), the conditional SEP has a closedform. For instance, in [2, Chap. 10] the SEP for square QAM 
is given by

$$
\begin{array}{r}
P_{S}(E)=4\left(1-\frac{1}{\sqrt{M}}\right) Q\left(\sqrt{\frac{3 E_{S}}{N_{0}(M-1)}}\right) \\
-4\left(1-\frac{1}{\sqrt{M}}\right)^{2} Q^{2}\left(\sqrt{\frac{3 E_{S}}{N_{0}(M-1)}}\right),
\end{array}
$$

where $E_{S}$ is the energy of the symbol, $M$ is the size of the constellation, and $Q($.$) is the Gaussian Q$ function, i.e. $Q(x)=$ $\int_{x}^{\infty} \frac{e^{-t^{2} / 2}}{\sqrt{2 \pi}} d t$

The generalized Gaussian distribution presented in (1). It has the same symmetric property as the Gaussian distribution and its corresponding complementary cumulative distribution function is the generalized Gaussian $Q$ function, $Q_{\alpha}($.$) , de-$ fined as [8]

$$
Q_{\alpha}(x)=\frac{\alpha \Lambda_{0}}{2 \Gamma(1 / \alpha)} \int_{x}^{\infty} \exp \left(-\Lambda_{0}^{\alpha}|u|^{\alpha}\right) d u .
$$

Similar to the standard Gaussian case, it can be easily shown that the conditional SEP for the M-QAM signalling over a fading channel with envelop $\mathcal{H}$ and subject to AWGGN, can simply be written as

$$
\begin{array}{r}
\operatorname{Pr}(e \mid \gamma)=4\left(1-\frac{1}{\sqrt{M}}\right) Q_{\alpha}\left(\sqrt{\frac{3 \gamma}{M-1}}\right) \\
-4\left(1-\frac{1}{\sqrt{M}}\right)^{2} Q_{\alpha}^{2}\left(\sqrt{\frac{3 \gamma}{M-1}}\right)
\end{array}
$$

where $\gamma=\frac{\mathcal{H}^{2} E_{\mathcal{S}}}{N_{0}}$ is the instantaneous SNR per symbol at the receiver.

The average SEP (ASEP) is obtained by averaging the conditional SEP (5) over the PDF of the SNR, $p_{\gamma}(\gamma)$, yielding

$$
\begin{aligned}
\operatorname{Pr}(e)= & \int_{0}^{\infty} \operatorname{Pr}(e \mid \gamma) p_{\gamma}(\gamma) d \gamma \\
= & \int_{0}^{\infty}\left[4\left(1-\frac{1}{\sqrt{M}}\right) Q_{\alpha}\left(\sqrt{\frac{3 \gamma}{M-1}}\right)\right. \\
& \left.-4\left(1-\frac{1}{\sqrt{M}}\right)^{2} Q_{\alpha}^{2}\left(\sqrt{\frac{3 \gamma}{M-1}}\right)\right] p_{\gamma}(\gamma) d \gamma
\end{aligned}
$$

This expression is valid for any type of fading, in particular for the EGK fading introduced in the next section and in which the integral form given in (6b) will be solved in closed-from using alternative expressions for $Q_{\alpha}($.$) and the PDF of the$ $\operatorname{SNR} p_{\gamma}(\gamma)$.

\section{AVERAGE SEP OVER EGK FADING}

In this paper, the fading is assumed to follow the EGK distribution which is a generalized fading distribution that captures many common fading distributions as special cases. According to this model, the PDF of the instantaneous SNR, derived from the channel envelope $\mathcal{H}$, is given in [7, Eq. (2)], as

$$
\begin{aligned}
p_{\gamma}(\gamma)= & \frac{\xi}{\Gamma\left(m_{s}\right) \Gamma(m)}\left(\frac{\beta_{s} \beta}{\bar{\gamma}}\right)^{m \xi} \gamma^{m \xi-1} \\
& \quad \times \Gamma\left(m_{s}-m \frac{\xi}{\xi_{s}}, 0,\left(\frac{\beta_{s} \beta}{\bar{\gamma}}\right)^{m \xi} \gamma^{\xi}, \frac{\xi}{\xi_{s}}\right)
\end{aligned}
$$

defined over $\gamma \in(0, \infty)$. In (7), the parameters $\bar{\gamma}=\mathbb{E}[\gamma]$ is the average SNR per QAM symbol, $m(0.5 \leq m<\infty)$ and $\xi(0 \leq \xi<\infty)$ represent the fading figure (diversity severity / order) and the fading shaping factor, respectively, while $m_{s}\left(0.5 \leq m_{s}<\infty\right)$ and $\xi_{s}\left(0 \leq \xi_{s}<\infty\right)$ represent the shadowing severity and the shadowing shaping factor (inhomogeneity), respectively. Furthermore, $\Gamma($.$) is the$ Gamma function, the parameters $\beta=\Gamma(m+1 / \xi) / \Gamma(m)$, $\beta_{s}=\Gamma\left(m_{s}+1 / \xi_{s}\right) / \Gamma\left(m_{s}\right)$, and $\Gamma(\cdot, \cdot, \cdot, \cdot)$ is the extended incomplete Gamma function defined in $[9$, Eq. (6.2)] as

$$
\Gamma(a, x, b, \beta)=\int_{x}^{\infty} r^{a-1} \exp \left(-r-b r^{-\beta}\right) d r,
$$

where $a, \beta, b \in \mathbb{C}$ and $x \in \mathbb{R}^{+}$.

To derive a closed-form expression of the SEP for EGK fading subjected to AWGGN, we need to use alternative expressions of the $Q_{\alpha}($.$) function in (4) and p_{\gamma}(\gamma)$ in (7). More specifically, expressing (4) and (7) in terms of the Fox $\mathrm{H}$ function (FHF) [10, Eq. (1.1.1)], [11], H. .[.], whose MATHEMATICA implementation is given in [12, Appendix], the integral in (6b) can be re-written as a sum of the product of two and three FHFs which can be solved in closed-form. For instance, we can write

$$
\begin{gathered}
p_{\gamma}(\gamma)=\frac{1}{\Gamma\left(m_{s}\right) \Gamma(m) \gamma} \mathrm{H}_{0,2}^{2,0}\left[\frac{\beta_{s} \beta}{\bar{\gamma} \gamma} \mid \begin{array}{c}
\left(m_{s}, \frac{1}{\xi_{s}}\right),\left(m, \frac{1}{\xi}\right) \\
\end{array},\right. \\
Q_{\alpha}(x)=\frac{1}{2 \Gamma(1 / \alpha)} \mathrm{H}_{1,2}^{2,0}\left[\Lambda_{0}^{\alpha}|x|^{\alpha} \mid \begin{array}{c}
(1,1) \\
\left(\frac{1}{\alpha}, 1\right),(0,1)
\end{array}\right] .
\end{gathered}
$$

By replacing (9) and (10) in (6b), we get two terms in integral form. The first term includes an integral of two FHFs, studied and solved in [8]. The second term contains an integral involving three FHFs (one from the EGK distribution in (9), and a squared one from the $Q_{\alpha}($.$) function in (10)). Using$ the identity [13, Eq. (2.3)], the second part can be expressed in terms of the FHF of two variables [13], known also as the

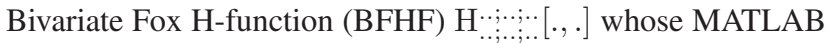
implementation is outlined in [14]. In conclusion, a closedform expression of the ASEP is obtained in (11).

It is important to note that this closed-form result (11) is a new generic expression for the ASEP of square M-QAM signaling over EGK fading channels subject to AWGGN. In the next section, we will discuss some special cases of noise and fading giving some simplified expressions. 


$$
\begin{aligned}
& \operatorname{Pr}(e)=\quad \frac{4\left(1-\frac{1}{\sqrt{M}}\right)}{\alpha \Gamma(1 / \alpha) \Gamma\left(m_{s}\right) \Gamma(m)} \mathrm{H}_{2,3}^{2,2}\left[\frac{\beta_{s} \beta(M-1)}{3 \Lambda_{0}^{2} \bar{\gamma}} \mid \begin{array}{c}
\left(1-\frac{1}{\alpha}, \frac{2}{\alpha}\right),\left(1, \frac{2}{\alpha}\right) \\
\left(m_{s}, \frac{1}{\xi_{s}}\right),\left(m, \frac{1}{\xi}\right),\left(0, \frac{2}{\alpha}\right)
\end{array}\right] \\
& -\frac{2\left(1-\frac{1}{\sqrt{M}}\right)^{2}}{\alpha \Gamma(1 / \alpha)^{2} \Gamma\left(m_{s}\right) \Gamma(m)} \mathrm{H}_{2,1 ; 0,2 ; 1,2}^{0,2,2,2,0}\left[\begin{array}{c|c}
\left(1-\frac{1}{\alpha} ; \frac{2}{\alpha}, 1\right),\left(1 ; \frac{2}{\alpha}, 1\right) & \\
\left(0 ; \frac{2}{\alpha}, 1\right) & \\
\left(m_{s}, \frac{1}{\xi_{s}}\right),\left(m, \frac{1}{\xi}\right) & \frac{\beta_{s} \beta(M-1)}{3 \Lambda_{0}^{2} \bar{\gamma}}, 1 \\
(1,1) &
\end{array}\right] .
\end{aligned}
$$

\section{Special Cases of Noise and Fading}

\section{A. EGK Fading with Additive Lapalacian Noise}

The Laplacian noise is a special case of the generalized Gaussian noise when $\alpha$ is set to 1 . In this case, and using the properties of the FHF [10], and BFHF functions [13], the general ASEP expression in (11) reduces to

$$
\begin{aligned}
\operatorname{Pr}(e)=\frac{4\left(1-\frac{1}{\sqrt{M}}\right)}{\Gamma\left(m_{s}\right) \Gamma(m)} \mathrm{H}_{1,2}^{2,1}\left[\frac{\beta_{s} \beta(M-1)}{6 \bar{\gamma}} \mid \begin{array}{c}
(1,2) \\
\left(m_{s}, \frac{1}{\xi_{s}}\right),\left(m, \frac{1}{\xi}\right)
\end{array}\right] & -\frac{2\left(1-\frac{1}{\sqrt{M}}\right)^{2}}{\Gamma\left(m_{s}\right) \Gamma(m)} \times \\
\mathrm{H}_{1,0 ; 0,2 ; 0,1}^{0,1 ; 2,0 ; 1,0}\left[\begin{array}{c|c}
(1 ; 2,1) \\
\left(m_{s}, \frac{1}{\left.\xi_{s}\right),\left(m, \frac{1}{\xi}\right)}\right.
\end{array}\right. & \left.\frac{\beta_{s} \beta(M-1)}{6 \bar{\gamma}}, 1\right] .
\end{aligned}
$$

This formula contains also the FHF and BFHF, but the advantage that in this case these functions have a lower rank than in the general expression given in (11), which reduces the complexity of the evaluation of these functions. Since the Laplacian noise is an interesting case of study, it is also of interest to study other special fading cases in conjunction with the Laplacian noise. For instance, considering the generalized Nakagami-m (GNM) fading by setting $\xi_{s}=1$ and $m_{s} \rightarrow \infty$ in (7), the ASEP in (12) further simplifies to

$$
\begin{aligned}
& \operatorname{Pr}(e)=\frac{4\left(1-\frac{1}{\sqrt{M}}\right)}{\Gamma(m)} \mathrm{H}_{1,1}^{1,1}\left[\begin{array}{c|c}
\frac{\beta(M-1)}{6 \bar{\gamma}} & (1,2) \\
\left(m, \frac{1}{\xi}\right)
\end{array}\right]
\end{aligned}
$$

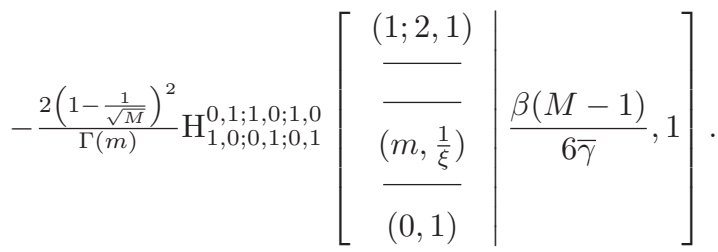

\section{B. Generalized $K(G K)$ Fading with AWGN}

We now consider the GK fading in conjunction with the classical Gaussian noise by setting $\alpha=2, \xi=1$, and $\xi_{s}=$ 1. With these settings, the general ASEP expression in (11) reduces to

$$
\begin{array}{r}
\operatorname{Pr}(e)=\frac{2\left(1-\frac{1}{\sqrt{M}}\right)}{\sqrt{\pi} \Gamma\left(m_{s}\right) \Gamma(m)} \mathrm{G}_{2,3}^{2,2}\left[\frac{2 m_{s} m(M-1)}{3 \bar{\gamma}} \mid \begin{array}{c}
\left(\frac{1}{2}, 1\right) \\
\left(m_{s}, m, 0\right)
\end{array}\right] \\
-\frac{\left(1-\frac{1}{\sqrt{M}}\right)^{2}}{\pi \Gamma\left(m_{s}\right) \Gamma(m)} \mathrm{G}_{2,1 ; 0,2 ; 1,2}^{0,2 ; 2,2,0}\left[\begin{array}{c|c}
\frac{\left(\frac{1}{2}, 1\right)}{(0)} & \frac{2 m_{s} m(M-1)}{3 \bar{\gamma}}, 1 \\
\left(m_{s}, m\right) & \frac{(1)}{\left(\frac{1}{2}, 0\right)}
\end{array}\right],
\end{array}
$$

where the first term is expressed in terms of the standard Meijer G-function (MGF) [10, Eq. (2.9.1)] which is a built in function in MATHEMATICA, while the second term is expressed in terms of the bivariate Meijer G-function (BMGF) [15] whose MATHEMATICA implementation is given in [16, Table II].

It is necessary to mention that in these special cases, we are not just setting the parameters in the general expression (11). Rather, in each case, we offered simplified closedform expressions of the ASEP with reduced computational complexity. However, the next two special cases involving the classical Rayleigh fading lead to further simplified expressions of the ASEP that include only common functions.

\section{Rayleigh Fading with Additive Laplacian Noise}

We have treated the Laplacian noise case and the corresponding ASEP is presented in (13). The Rayleigh fading case is obtained by setting $m=1$ and $\xi=1$ in (13), yielding

$$
\begin{gathered}
\operatorname{Pr}(e)=4\left(1-\frac{1}{\sqrt{M}}\right) \mathrm{H}_{1,1}^{1,1}\left[\frac{M-1}{6 \bar{\gamma}} \mid \begin{array}{c}
(1,2) \\
(1,1)
\end{array}\right] \\
-2\left(1-\frac{1}{\sqrt{M}}\right)^{2} \mathrm{H}_{1,0 ; 0,1 ; 0,1}^{0,1 ; 0 ; 1,0}\left[\frac{\overline{(1 ; 2,1)}}{\frac{(1,1)}{(0,1)}} \mid \frac{M-1}{6 \bar{\gamma}}, 1\right] .
\end{gathered}
$$


Using some properties of the FHF [10], and BFHF [17], it can be shown that (15) reduces to

$$
\begin{aligned}
& \operatorname{Pr}(e)= \\
& \quad 2\left(1-\frac{1}{\sqrt{M}}\right)\left(1-\sqrt{\frac{6 \pi \bar{\gamma}}{M-1}} e^{\frac{1.5 \bar{\gamma}}{M-1}} Q\left(\sqrt{\frac{3 \bar{\gamma}}{M-1}}\right)\right) \\
& -\left(1-\frac{1}{\sqrt{M}}\right)^{2}\left(1-2 \sqrt{\frac{6 \pi \bar{\gamma}}{M-1}} e^{\frac{6 \bar{\gamma}}{M-1}} Q\left(\sqrt{\frac{12 \bar{\gamma}}{M-1}}\right)\right),
\end{aligned}
$$

which is a very simple expression involving only the standard Gaussian $Q$ function (i.e. $Q(x)=Q_{2}(x)$ ). It should be noted that, to our best knowledge, (16) is a new expression not reported previously.

\section{Rayleigh Fading with AWGN}

The AWGN was studied in the second special case and an expression of the ASEP of M-QAM over GK fading with AWGN was derived and given in (14). The Rayleigh fading is a special case of the GK distribution by simply setting $m=1$ and $m_{s} \rightarrow \infty$. In this case, (14) reduces to

$$
\begin{aligned}
& \operatorname{Pr}(e)=\frac{2\left(1-\frac{1}{\sqrt{M}}\right)}{\sqrt{\pi}} \mathrm{G}_{2,2}^{1,2}\left[\frac{2(M-1)}{3 \bar{\gamma}} \mid \begin{array}{c}
\left(\frac{1}{2}, 1\right) \\
(1,0)
\end{array}\right] \\
& -\frac{\left(1-\frac{1}{\sqrt{M}}\right)^{2}}{\pi} \mathrm{G}_{2,1 ; 0,1 ; 1,2}^{0,2 ; 1,0 ; 2,0}\left[\begin{array}{c|c}
\left(\frac{1}{2}, 1\right) & \\
\frac{(0)}{(1)} & \left.\frac{2(M-1)}{3 \bar{\gamma}}, 1\right] \\
(1) &
\end{array}\right] \text {. }
\end{aligned}
$$

The expression in (17) involves reduced rank MGF and BMGF functions in comparison with (14). However, more interestingly, using some properties of these special functions [17] and extra manipulations, it can be shown that this formula simplifies to

$$
\begin{gathered}
\operatorname{Pr}(e)=2\left(1-\frac{1}{\sqrt{M}}\right)\left(1-\sqrt{\frac{3 \bar{\gamma}}{2(M-1)+3 \bar{\gamma}}}\right) \\
-\left(1-\frac{1}{\sqrt{M}}\right)^{2} \times \\
{\left[1-\sqrt{\frac{3 \bar{\gamma}}{2(M-1)+3 \bar{\gamma}}}\left(\frac{4}{\pi} \tan ^{-1} \sqrt{\frac{2(M-1)+3 \bar{\gamma}}{3 \bar{\gamma}}}\right)\right],}
\end{gathered}
$$

where $\tan ^{-1}($.$) is the inverse tangent function, in agreement$ with the known expression [1, Chap. 8, Eq. (8.106)] of the ASEP of M-QAM over Rayleigh fading and subject to AWGN.

\section{Numerical RESUlTS}

In this section, we plot the ASEP of 16-QAM signaling for different type of noise (i.e. different values of $\alpha$ ), and fading (i.e. different values of $m, m_{s}, \xi$, and $\xi_{s}$ ). As a first numerical example, we look into Gaussian and Laplacian

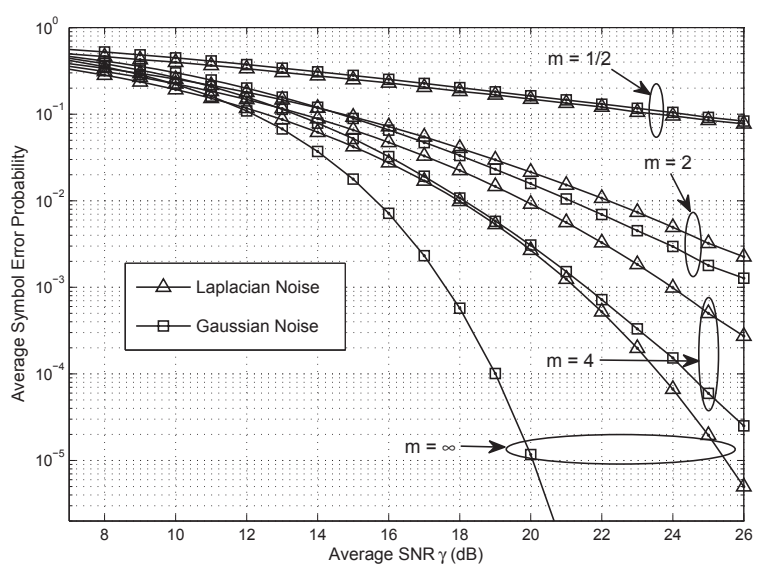

Fig. 1. ASEP of 16-QAM over Nakagami- $m$ fading subject to Laplacian noise and Gaussian noise. The markers denote simulation results while solid lines represent analytical results.

noise in conjunction with Nakagami- $m$ fading. The Nakagmi$\mathrm{m}$ fading is obtained by setting the fading shaping factor $\xi=1$ in (13) for the Laplacian noise, since the Nakagami-m fading is a special case of the GNM. The numerical results of this example are presented in Fig. 1 for four values of the Nakagami-m fading parameter, namely $m=\frac{1}{2}, 2,4, \infty$. The numerical results obtained from the main formula (13) are compared to computer-based Monte-Carlo simulation results. It is clear that the numerical results and simulation results match perfectly as a validation of our analytical results. In both noise cases, the ASEP improves as the fading parameter $m$ increases, as expected. In addition, for a high amount of fading (i.e. $m=\frac{1}{2}$ ), the ASEP for the Laplacian case is better than the ASEP for the Gaussian case. However, for a low amount of fading (i.e. $m \geq 2$ ), we note that there are two regimes. The first regime is for low SNR $(\bar{\gamma} \leq 12 \mathrm{~dB})$ in which the Laplacian noise has better performance than the Gaussian noise, and in second regime, for high SNR $(\bar{\gamma}>12 \mathrm{~dB})$ in which ASEP for the Gaussian case is better than the ASEP for the Laplacian case.

In Fig. 2, we look into the Rayleigh fading case and vary the noise parameter $\alpha$. In this case the ASEP can be re-written from (11) as

$$
\begin{aligned}
& \operatorname{Pr}(e)=\frac{4\left(1-\frac{1}{\sqrt{M}}\right)}{\alpha \Gamma(1 / \alpha)} \mathrm{H}_{2,2}^{1,2}\left[\frac{M-1}{3 \Lambda_{0}^{2} \bar{\gamma}} \mid \begin{array}{c}
\left(1-\frac{1}{\alpha}, \frac{2}{\alpha}\right),\left(1, \frac{2}{\alpha}\right) \\
\left(m, \frac{1}{\xi}\right),\left(0, \frac{2}{\alpha}\right)
\end{array}\right] \\
& -\frac{2\left(1-\frac{1}{\sqrt{M}}\right)^{2}}{\alpha \Gamma(1 / \alpha)^{2}} \times \\
& \mathrm{H}_{2,1 ; 0,1 ; 1,2}^{0,2 ; 1,0 ; 2,0}\left[\begin{array}{c|c}
\left(1-\frac{1}{\alpha} ; \frac{2}{\alpha}, 1\right),\left(1 ; \frac{2}{\alpha}, 1\right) & \\
\left(0 ; \frac{2}{\alpha}, 1\right) & \frac{M-1}{3 \Lambda_{0}^{2} \bar{\gamma}}, 1 \\
\left(m, \frac{1}{\xi}\right) & \\
(1,1) &
\end{array}\right] .
\end{aligned}
$$




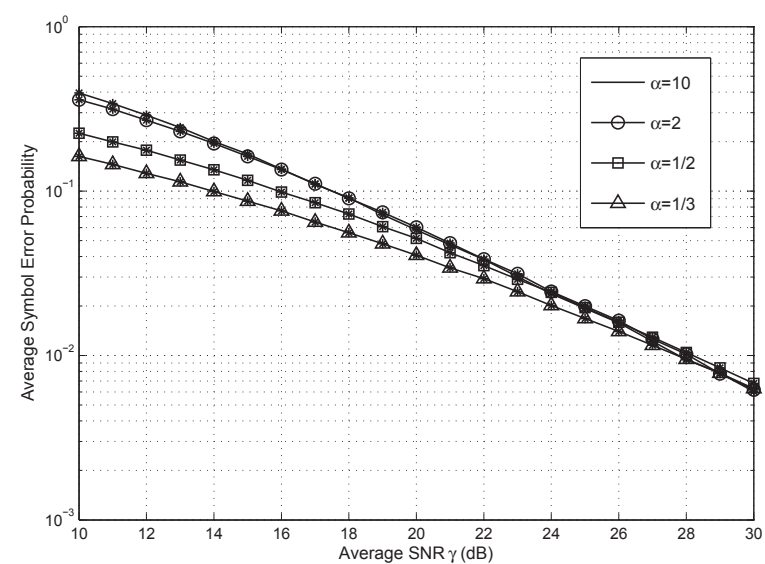

Fig. 2. ASEP of 16-QAM over Rayleigh fading subject to generalized Gaussian noise (the markers denote simulation results while solid lines represent analytical results).

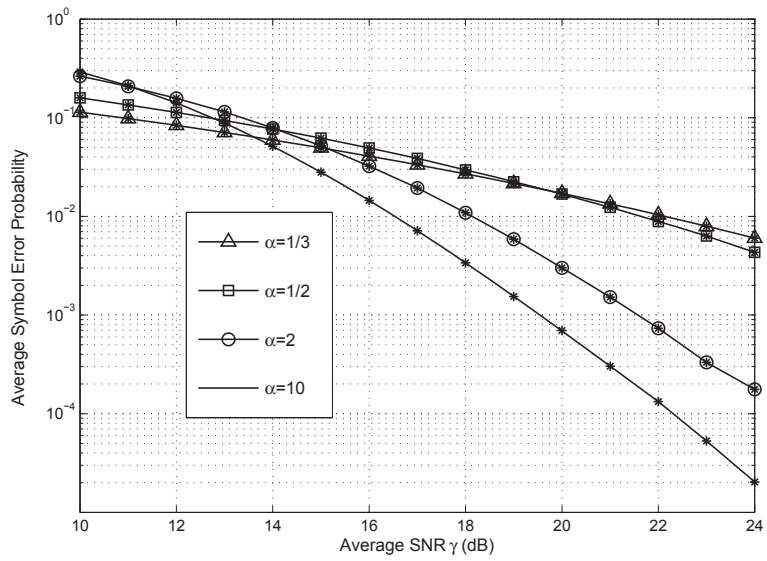

Fig. 3. ASEP of 16-QAM over Nakagami-4 fading subject to generalized Gaussian noise (the markers denote simulation results while solid lines represent analytical results).

Note again from Fig. 2 that the simulation results match perfectly the analytical results obtained from (19). In this case of Rayleigh fading, it is worth mentioning that the system has better performance by decreasing the noise parameter $\alpha$, which confirms the result found in the previous example when the Laplacian noise has better performance than the Gaussian noise for high amount of fading. At high SNR $(\bar{\gamma}>28 \mathrm{~dB})$, the performance of the system converges essentially to one curve for different values of noise parameter.

Finally, in order to see the impact of the fading parameter, we draw the ASEP for the case of Nakagami-4 fading and the same values of the noise parameter in Fig. 3. Similar to what happened in the first example, we have also in this case two regions. For low SNR the ASEP decreases with the noise parameter. However, for high SNR, the system performance gets better by increasing $\alpha$.

\section{CONCLUSion}

In this paper, we studied the average symbol error probability of square M-QAM signaling when transmitted over an extended generalized-K fading subject to additive generalized Gaussian noise. A closed-form of the ASEP was derived in terms of the FHF and BFHF functions. To illustrate the mathematical formalism, we presented some numerical example for the Nakagami-m fading and Rayleigh fading. The analytical results were validated by numerical and simulation results and the impact of the generalized Gaussian noise and generalized fading parameters on the ASEP was studied.

\section{REFERENCES}

[1] M. K. Simon and M.-S. Alouini, Digital Communication Over Fading Channels, 1st ed. New York: Wiley, 2005.

[2] M. K. Simon, S. M. Hinedi, and W. C. Lindsey, Digital Communication Techniques: Signal Design And Detection. Upper Saddle River, NJ: Prentice Hall, 1995.

[3] J. Lu, K. B. Letaief, J. C.-I. Chuang, and M. L. Liou, "M-PSK and M-QAM BER computation using signal-space concepts," IEEE Transactions on Communications, vol. 47, pp. 181-184, February 1999.

[4] X. Yang, M. Li, and X. Zhao, "Optimum detectors of line spectrum signals in generalized Gaussian noise," in Proceedings of the Third International Conference on Measuring Technology and Mechatronics Automation (ICMTMA'2011), Sanya, China, Jan. 2011, pp. 819-822.

[5] R. Viswanathan and A. Ansari, "Distributed detection of a signal in generalized Gaussian noise," IEEE Transcations on Acoustics, Speech and Signal Processing, vol. 37, no. 5, pp. 775-778, May 1989.

[6] S. Zahabi and A. Tadaion, "Local spectrum sensing in non-Gaussian noise," in Proceedings of the IEEE 17th International Conference on Telecommunication (ICT'2010), Doha, Qatar, April 2010, pp. 843-847.

[7] F. Yilmaz and M.-S. Alouini, "A new simple model for composite fading channels: Second order statistics and channel capacity," in IEEE 7th International Symposium on Wireless Communication Systems (ISWCS'2010), York, UK, Sept. 2010, pp. 676-680.

[8] H. Soury, F. Yilmaz, and M.-S. Alouini, "Average bit error probability of binary coherent signaling over generalized fading channels subject to additive generalized Gaussian noise," IEEE Communications Letters, vol. 16, no. 6, pp. 785-788, June 2012.

[9] M. A. Chaudhry and S. M. Zubair, On a Class of Incomplete Gamma Function with Applications. Boca Raton-London-Ney YorkWashington, D.C.: Chapman \& Hall/CRC, 2002.

[10] A. Kilbas and M. Saigo, H-Transforms : Theory and Applications (Analytical Method and Special Function), 1st ed. CRC Press, 2004.

[11] A. P. Prudnikov, Y. A. Brychkov, , and O. I. Marichev, Integral and Series: Volume 3, More Special Functions. CRC Press Inc., 1990.

[12] F. Yilmaz and M.-S. Alouini, "Product of the powers of generalized Nakagami-m variates and performance of cascaded fading channels," in Proceedings of the IEEE Global Telecommunication Conference (GLOBECOM'2009), Honolulu, Hawai, USA, Dec 2009, pp. 1-8.

[13] P. Mittal and K. Gupta, "An integral involving generalized function of two variables," in Indian Acad. Sci., 1972, pp. 117-123.

[14] K. Peppas, "A new formula for the average bit error probability of dualhop amplify-and-forward relaying systems over generalized shadowed fading channels," IEEE Wireless Communications Letters, vol. 1, no. 2, pp. 85-88, April 2012

[15] M. Shah, "On generalization of some results and their applications," Collectamea Mathematica, vol. 24, no. 3, pp. 249-266, 1973.

[16] I. Ansari, S. Al-Ahmadi, F. Yilmaz, M.-S. Alouini, and H. Yanikomeroglu, "A new formula for the BER of binary modulations with dual-branch selection over generalized-K composite fading channels," IEEE Transactions on Communications, vol. 59, no. 10, pp. 2654-2658, October 2011.

[17] H. M. Srivastava, K. C. Gupta, and S. P. Goyal, The H-functions of One and Two Variables, with Applications. South Asian Publishers, 1982. 